\title{
On the connection between discrete linear repetitive processes and 2-D discrete linear systems
}

\author{
M. S. Boudellioua ${ }^{1} \cdot$ K. Galkowski ${ }^{2}$ (D) E. Rogers ${ }^{3}$
}

Received: 12 February 2016 / Revised: 12 July 2016 / Accepted: 9 September 2016 / Published online: 19 September 2016

(c) The Author(s) 2016. This article is published with open access at Springerlink.com

\begin{abstract}
A direct method is developed that reduces a polynomial system matrix describing a discrete linear repetitive process to a 2- $D$ singular state-space form such that all the relevant properties, including the zero structure of the system matrix, are retained. It is shown that the transformation linking the original polynomial system matrix with its associated 2- $D$ singular form is zero coprime system equivalence. The exact nature of the resulting system matrix in singular form and the transformation involved are established.
\end{abstract}

Keywords Linear repetitive processes $\cdot 2-D$ discrete systems $\cdot$ System matrix $\cdot 2-D$ singular form $\cdot$ Zero-coprime system equivalence $\cdot$ Invariant zeros

\section{Introduction}

State-space models play an important role in the theory of 1- $D$ finite dimensional linear systems. Over the last decades there has been research on extending the state-space representation to more general systems, e.g. time-delay systems or systems described by partial differential equations. Another extension from 1- $D$ to 2- $D$ is the discrete linear state-space model that has a number of variants, e.g., Roesser (1975), Attasi (1973) or Fornasini and Marchesini (1976).

K. Galkowski

k.galkowski@issi.uz.zgora.pl

M. S. Boudellioua

boudell@squ.edu.om

E. Rogers

etar@ecs.soton.ac.uk

1 Department of Mathematics and Statistics, Sultan Qaboos University, Muscat, Oman

2 Institute of Control and Computation Engineering, University of Zielona Gora, Zielona Gora, Poland

3 Department of Electronics and Computer Science, University of Southampton, Southampton SO17 1BJ, UK 
Several authors have proposed a generalized state-space description for 2- $D$ systems, e.g., Zak (1984) based on the Roesser model and Kaczorek (1988) based on the Fornasini-Marchesini model. Also Galkowski (2001) developed an algorithm for reducing $2-D$, and in general $n-D(n \geq 2)$, rational function matrices to the singular and nonsingular Roesser forms. More recent work on this approach includes, e.g., Li et al. (2012) and references therein.

In this paper, a direct method for the reduction of a polynomial matrix description arising from linear repetitive processes Rogers et al. (2007) to an equivalent 2- $D$ singular state-space system is developed. The exact structure of the resulting system matrix in singular form is given and the transformation linking it to the original system matrix established. It is shown that this transformation is zero-coprime-system-equivalence. This type of equivalence has been studied by many authors, e.g., Levy (1981), Johnson (1993) and Pugh et al. (1996, 1998). In particular, Pugh et al. (1998) have shown that zero-coprime system equivalence forms the basis of the connection between all least order polynomial realizations of a given 2- $D$ transfer function matrix. Also, state-space models of discrete linear repetitive processes have been transformed to the equivalent Roesser or Fornasini-Marchesini forms, see, e.g., Galkowski et al. (1999); Rogers et al. (2007) and this is one route to characterize systems properties, such as local reachability/controllability (Galkowski et al. 1998), for these processes.

Throughout this paper the dimensions of the zero and identity matrices are specified only when essential to the analysis. In all other cases, these matrices are denoted by 0 and $I$ respectively.

\section{Discrete linear repetitive processes}

Following Rogers et al. (2007), the state-space model of a discrete linear repetitive process has the following form over the two indeterminates $p$ and $k$ where $0 \leq p \leq \alpha-1, k \geq 0$

$$
\begin{aligned}
x_{k+1}(p+1) & =A x_{k+1}(p)+B_{0} y_{k}(p)+B u_{k+1}(p) \\
y_{k+1}(p) & =C x_{k+1}(p)+D 0 y_{k}(p)+D u_{k+1}(p)
\end{aligned}
$$

In this model $\alpha<+\infty$ denotes the finite pass length and on pass $k \geq 0 x_{k}(p) \in \mathbb{R}^{n}$ is the state vector, $y_{k}(p) \in \mathbb{R}^{m}$ is the pass profile vector, which also serves as a system output, and $u_{k}(p) \in \mathbb{R}^{l}$ is the input vector.

To complete the process description, it is necessary to specify the boundary conditions, i.e., the state initial vector on each pass and the initial pass profile (i.e. on the 0th pass). For the purposes of this paper, it is assumed that the state initial vector at the start of each new pass is of the form $x_{k+1}(0)=d_{k+1}, k \geq 0$ and $y_{0}(p)=f(p), 0 \leq p \leq \alpha-1$, where the $n \times 1$ vector $d_{k+1}$ has known constant entries and those in the $m \times 1$ vector $f(p)$ are known functions of $p$.

A heavily used state-space model for the analysis of discrete linear systems recursive over the upper right quadrant of the 2- $D$ plane was first proposed by Roesser (1975). In this model a state vector is defined for vertical and horizontal axes independently. Denoting these vectors by $x^{h}(i, j) \in \mathbb{R}^{n}$ and $x^{v}(i, j) \in \mathbb{R}^{m}$, and also introducing the output vector $y(i, j) \in \mathbb{R}^{q}$ and the input vector $u(i, j) \in \mathbb{R}^{l}$, the Roesser state-space model has the form

$$
\begin{aligned}
{\left[\begin{array}{l}
x^{h}(i+1, j) \\
x^{v}(i, j+1)
\end{array}\right] } & =\left[\begin{array}{ll}
A_{11} & A_{12} \\
A_{21} & A_{22}
\end{array}\right]\left[\begin{array}{l}
x^{h}(i, j) \\
x^{v}(i, j)
\end{array}\right]+\left[\begin{array}{l}
B_{1} \\
B_{2}
\end{array}\right] u(i, j) \\
y(i, j) & =\left[\begin{array}{ll}
C_{1} & C_{2}
\end{array}\right]\left[\begin{array}{l}
x^{h}(i, j) \\
x^{v}(i, j)
\end{array}\right]+D u(i, j)
\end{aligned}
$$


In this case boundary conditions are defined as $x^{h}(0, j)=f(j), j \geq 0$ and $x^{v}(i, 0)=$ $d(i), i \geq 0$, where the $n \times 1$ vector $f(j)$ and the $m \times 1$ vector $d(i)$ have known constant entries.

Another commonly used state-space model for 2- $D$ systems is the Fornasini Marchesini model (Fornasini and Marchesini 1976), where the state vector is not split into horizontal and vertical components and the structure is

$$
\begin{gathered}
z(i+1, j+1)=A_{1} z(i+1, j)+A_{2} z(i, j+1)+A_{3} z(i, j) \\
+B_{1} u(i+1, j)+B_{2} u(i, j+1) \\
y(i, j)=C z(i, j)+D u(i, j)
\end{gathered}
$$

where the vectors $z, y$ and $u$ are the (appropriately dimensioned) state, output and input vectors, respectively, with boundary conditions to be specified.

The Roesser model (2) and the discrete linear repetitive process model (1) are very similar but there are essential differences. The first is that repetitive processes are defined over a finite strip, or subset, the upper right quadrant of the 2- $D$ plane. These processes make a series of sweeps, or passes, through dynamics defined over the finite pass length. Once each pass is complete, the process resets to the starting location and the next pass can begin, either immediately after the resetting is complete or after a further period of time has elapsed. In a repetitive process the 2- $D$ systems structure arises from the influence of the previous pass profile on the current pass state and pass profile vectors, i.e., from the terms $B_{0} y_{k}(p)$ and $D_{0} y_{k}(p)$ in (1) respectively.

\section{System equivalence}

The concept of a polynomial system matrix was first introduced by Rosenbrock (1970) for standard, or 1- $D$, linear systems. The natural generalization to $2-D$ linear systems is the polynomial system description:

$$
\begin{aligned}
T\left(z_{1}, z_{2}\right) x & =U\left(z_{1}, z_{2}\right) u \\
y & =V\left(z_{1}, z_{2}\right) x+W\left(z_{1}, z_{2}\right) u
\end{aligned}
$$

where $x \in \mathbb{R}^{n}$ is the state vector, $u \in \mathbb{R}^{p}$ is the input vector and $y \in \mathbb{R}^{m}$ is the output vector, $T, U, V$ and $W$ are polynomial matrices with elements in $\mathbb{R}\left[z_{1}, z_{2}\right]$ of dimensions $r \times r, r \times p, m \times l$ and $m \times p$ respectively. The operators $z_{1}$ and $z_{2}$ may have various meanings depending on the type of system. For example, in delay-differential systems $z_{1}$ may represent a differential operator and $z_{2}$ a delay-operator. For 2- $D$ discrete systems, $z_{1}$ and $z_{2}$ represent horizontal and vertical shift operators, respectively and is the only case considered in this paper. The system (4) gives rise to the system matrix in the general form:

$$
P\left(z_{1}, z_{2}\right)=\left[\begin{array}{cc}
T\left(z_{1}, z_{2}\right) & U\left(z_{1}, z_{2}\right) \\
-V\left(z_{1}, z_{2}\right) & W\left(z_{1}, z_{2}\right)
\end{array}\right]
$$

where

$$
P\left(z_{1}, z_{2}\right)\left[\begin{array}{c}
x \\
-u
\end{array}\right]=\left[\begin{array}{c}
0 \\
-y
\end{array}\right]
$$

If $T\left(z_{1}, z_{2}\right)$ is invertible, the system matrix in (5) is said to be regular. In such cases, the transfer-function matrix corresponding to the system matrix in (5) is given by:

$$
G\left(z_{1}, z_{2}\right)=V\left(z_{1}, z_{2}\right) T^{-1}\left(z_{1}, z_{2}\right) U\left(z_{1}, z_{2}\right)+W\left(z_{1}, z_{2}\right)
$$


A special case of (5) is obtained for systems described by the following 2- $D$ singular general (SG) state-space model as given by Kaczorek (1988),

$$
\begin{array}{r}
\operatorname{Ex}(i+1, j+1)=A_{1} x(i+1, j)+A_{2} x(i, j+1) \\
+A_{0} x(i, j)+B_{1} u(i+1, j) \\
+B_{2} u(i, j+1)+B_{0} u(i, j) \\
y(i, j)=C x(i, j)+D u(i, j)
\end{array}
$$

where $x(i, j)$ is the state vector, $u(i, j)$ is the input vector, $y(i, j)$ is the output vector, $E$, $A_{0}, A_{1}, A_{2}, B_{0}, B_{1}, B_{2}, C$ and $D$ are constant real matrices of compatible dimensions and $E$ may be singular. Introduce the forward shift operators $z_{1}$ and $z_{2}$ in the horizontal and vertical directions, i.e.

$$
z_{1} x(i, j)=x(i+1, j), \quad z_{2} x(i, j)=x(i, j+1)
$$

respectively. Then the process dynamics described by (8) can be written in polynomial matrix description form as:

$$
P_{S G}\left(z_{1}, z_{2}\right)\left[\begin{array}{c}
x \\
-u
\end{array}\right]=\left[\begin{array}{c}
0 \\
-y
\end{array}\right]
$$

where over $\mathbb{R}\left[z_{1}, z_{2}\right]$,

$$
P_{S G}\left(z_{1}, z_{2}\right)=\left[\begin{array}{c|c}
z_{1} z_{2} E-z_{1} A_{1}-z_{2} A_{2}-A_{0} & z_{1} B_{1}+z_{2} B_{2}+B_{0} \\
\hline-C & D
\end{array}\right]
$$

is the system matrix of (8). If the matrix $z_{1} z_{2} E-z_{1} A_{1}-z_{2} A_{2}-A_{0}$ is invertible, the transfer-function matrix for such examples is:

$$
G_{S G}\left(z_{1}, z_{2}\right)=C\left(z_{1} z_{2} E-z_{1} A_{1}-z_{2} A_{2}-A_{0}\right)^{-1}\left(z_{1} B_{1}+z_{2} B_{2}+B_{0}\right)+D
$$

A singular Roesser type model (SR) has the state-space model:

$$
\begin{gathered}
E\left[\begin{array}{l}
x_{1}(i+1, j) \\
x_{2}(i, j+1)
\end{array}\right]=\left[\begin{array}{ll}
A_{11} & A_{12} \\
A_{21} & A_{22}
\end{array}\right]\left[\begin{array}{l}
x_{1}(i, j) \\
x_{2}(i, j)
\end{array}\right]+\left[\begin{array}{l}
B_{1} \\
B_{2}
\end{array}\right] u(i, j) \\
y(i, j)=\left[\begin{array}{ll}
C_{1} & C_{2}
\end{array}\right]\left[\begin{array}{l}
x_{1}(i, j) \\
x_{2}(i, j)
\end{array}\right]+\operatorname{Du}(i, j)
\end{gathered}
$$

where the matrix $E$ is singular. On employing $z_{1}$ and $z_{2}$ defined above, (13) can be represented by the polynomial equations:

$$
P_{S R}\left(z_{1}, z_{2}\right)\left[\begin{array}{c}
x_{1} \\
x_{2} \\
-u
\end{array}\right]=\left[\begin{array}{c}
0 \\
0 \\
-y
\end{array}\right]
$$

where

$$
P_{S R}\left(z_{1}, z_{2}\right)=\left[\begin{array}{c|c}
z_{1} E_{1}+z_{2} E_{2}-A & B \\
\hline-C & D
\end{array}\right]
$$

with $E=\left[\begin{array}{ll}E_{11} & E_{12} \\ E_{21} & E_{22}\end{array}\right], E_{1}=\left[\begin{array}{ll}E_{11} & 0 \\ E_{21} & 0\end{array}\right], E_{2}=\left[\begin{array}{ll}0 & E_{12} \\ 0 & E_{22}\end{array}\right], B^{T}=\left[\begin{array}{ll}B_{1}^{T} & B_{2}^{T}\end{array}\right]$ and $C=\left[\begin{array}{ll}C_{1} & C_{2}\end{array}\right]$ is the system matrix associated with (13). If the matrix $\left[z_{1} E_{1}+z_{2} E_{2}-A\right]$ is invertible, the transfer-function matrix corresponding to (15) is:

$$
G_{S R}\left(z_{1}, z_{2}\right)=C\left[z_{1} E_{1}+z_{2} E_{2}-A\right]^{-1} B+D
$$

One reason to analyze the singular Roesser model arises in the definition and characterization of controllability (or reachability but here this concept is not discussed further) for 
discrete linear repetitive processes, where more than one distinct concept can be formulated with a well defined physical meaning. In some applications for repetitive processes, such as material rolling, it may be required to produce a specified pass profile on a specified pass number (variations are possible such as not a-priori specifying the pass number but requiring that the specified pass profile is produced on some pass). A refinement of this property is local controllability where it is required that the pass profile has a pre-specified value at a particular point along a specified pass (again variations are possible).

In Galkowski et al. (1998) it was shown that conditions for local controllability of discrete linear repetitive processes can be obtained by converting the defining state-space model to that of a singular 2- $D$ Roesser state-space model. Hence applications driven interest in the links between the underlying descriptions that forms the motivation for the rest of this paper. Next some known concepts used in this paper are stated.

Definition 1 Let $\mathbb{T}(m, n)$ denote the class of $(r+m) \times(r+n)$ 2-D polynomial matrices, where $r>-\min (m, n)$. The subset, $\mathbb{P}(m, n)$ of $\mathbb{T}(m, n)$ obtained by requiring $r>0$ represents the 2- $D$ polynomial system matrices. Two polynomial system matrices $P_{1}\left(z_{1}, z_{2}\right)$ and $P_{2}\left(z_{1}, z_{2}\right)$ are said to be zero coprime equivalent if there exist polynomial matrices $S_{1}\left(z_{1}, z_{2}\right)$ and $S_{2}\left(z_{1}, z_{2}\right)$ of compatible dimensions such that

$$
S_{1}\left(z_{1}, z_{2}\right) P_{2}\left(z_{1}, z_{2}\right)=P_{1}\left(z_{1}, z_{2}\right) S_{2}\left(z_{1}, z_{2}\right)
$$

where $P_{1}\left(z_{1}, z_{2}\right)$ and $S_{1}\left(z_{1}, z_{2}\right)$ are zero left coprime and $P_{2}\left(z_{1}, z_{2}\right)$ and $S_{2}\left(z_{1}, z_{2}\right)$ are zero right coprime.

Pugh et al. (1996) and Pugh and El-Nabrawy (2003) have shown that zero coprime equivalence exhibits fundamental algebraic properties amongst its invariants. A basic transformation proposed for the study of 2-D system matrices by Levy (1981) and Johnson (1993) is zero coprime system equivalence. This transformation is characterized by the following definition.

Definition 2 Two polynomial system matrices $P_{1}\left(z_{1}, z_{2}\right)$ and $P_{2}\left(z_{1}, z_{2}\right) \in \mathbb{P}(m, n)$, are said to be zero coprime system equivalent if they are related by

$$
\underbrace{\left[\begin{array}{cc}
M\left(z_{1}, z_{2}\right) & 0 \\
X\left(z_{1}, z_{2}\right) & I_{m}
\end{array}\right]}_{S_{1}\left(z_{1}, z_{2}\right)} \underbrace{\left[\begin{array}{cc}
T_{1}\left(z_{1}, z_{2}\right) & U_{1}\left(z_{1}, z_{2}\right) \\
-V_{1}\left(z_{1}, z_{2}\right) & W_{1}\left(z_{1}, z_{2}\right)
\end{array}\right]}_{P_{2}\left(z_{1}, z_{2}\right)}=\underbrace{\left[\begin{array}{cc}
T_{2}\left(z_{1}, z_{2}\right) & U_{2}\left(z_{1}, z_{2}\right) \\
-V_{2}\left(z_{1}, z_{2}\right) & W_{2}\left(z_{1}, z_{2}\right)
\end{array}\right]}_{P_{1}\left(z_{1}, z_{2}\right)} \underbrace{\left[\begin{array}{cc}
N\left(z_{1}, z_{2}\right) Y\left(z_{1}, z_{2}\right) \\
I_{n}
\end{array}\right]}_{S_{2}\left(z_{1}, z_{2}\right)}
$$

where $P_{1}\left(z_{1}, z_{2}\right)$ and $S_{1}\left(z_{1}, z_{2}\right)$ are zero left coprime, $P_{2}\left(z_{1}, z_{2}\right)$ and $S_{2}\left(z_{1}, z_{2}\right)$ are zero right coprime and $M\left(z_{1}, z_{2}\right), N\left(z_{1}, z_{2}\right), X\left(z_{1}, z_{2}\right)$ and $Y\left(z_{1}, z_{2}\right)$ are polynomial matrices of compatible dimensions.

The transformation of zero coprime system equivalence plays a key role in certain aspects of 2-D systems theory. Moreover, this is an extension of Fuhrmann's strict system equivalence from the 1- $D$ to the 2- $D$ setting and has been shown by Levy (1981), Johnson (1993) and Pugh et al. $(1996,1998)$ to preserve important properties of the system matrix $P\left(z_{1}, z_{2}\right)$.

Lemma 1 (Johnson (1993)) The transformation of zero coprime system equivalence preserves the transfer-function matrix and the zero structure of the matrices:

$$
T_{i}\left(z_{1}, z_{2}\right), \quad P_{i}\left(z_{1}, z_{2}\right), \quad\left[T_{i}\left(z_{1}, z_{2}\right) U_{i}\left(z_{1}, z_{2}\right)\right], \quad\left[\begin{array}{c}
T_{i}\left(z_{1}, z_{2}\right) \\
-V_{i}\left(z_{1}, z_{2}\right)
\end{array}\right], \quad i=1,2 .
$$




\section{System matrices of linear repetitive processes}

In this section linear repetitive processes described by (1) are considered. Introduce the forward shift operators $z_{1}$ in the pass-to-pass direction and $z_{2}$ in the along the pass direction, i.e.,

$$
z_{1} s_{k}(p)=s_{k+1}(p), \quad z_{2} s_{k}(p)=s_{k}(p+1)
$$

respectively, where the signal $s_{k}(p)$ represents $x_{k}(p)$ or $y_{k}(p)$ as appropriate. The process dynamics can now be written in polynomial matrix form as:

$$
P_{R P}\left(z_{1}, z_{2}\right)\left[\begin{array}{c}
x_{k}(p) \\
\frac{y_{k}(p)}{-u_{k}(p)}
\end{array}\right]=\left[\begin{array}{c}
0 \\
0 \\
\hline-y_{k}(p)
\end{array}\right]
$$

where

$$
P_{R P}\left(z_{1}, z_{2}\right)=\left[\begin{array}{cc|c}
z_{1} z_{2} I_{n}-z_{1} A & -B_{0} & z_{1} B \\
-z_{1} C & z_{1} I_{m}-D_{0} & z_{1} D \\
\hline 0_{m, n} & -I_{m} & 0_{m, p}
\end{array}\right]
$$

is the system matrix associated with (1). Alternatively the dynamics of (1) can be represented in transfer-function matrix form as:

$$
Y\left(z_{1}, z_{2}\right)=G_{R P}\left(z_{1}, z_{2}\right) U\left(z_{1}, z_{2}\right)
$$

where

$$
G_{R P}\left(z_{1}, z_{2}\right)=\left[\begin{array}{ll}
0 & I_{m}
\end{array}\right]\left[\begin{array}{cc}
z_{1} z_{2} I-z_{1} A & -B_{0} \\
-z_{1} C & z_{1} I-D_{0}
\end{array}\right]^{-1}\left[\begin{array}{c}
z_{1} B \\
z_{1} D
\end{array}\right]
$$

Example 1 Consider the discrete repetitive process with state-space model matrices:

$$
\begin{gathered}
A=\left[\begin{array}{cc}
1 & -1 \\
2 & 0
\end{array}\right], \quad B_{0}=\left[\begin{array}{ll}
0 & 1 \\
2 & 1
\end{array}\right], \quad B=\left[\begin{array}{ll}
1 & 0 \\
1 & 1
\end{array}\right] \\
C=\left[\begin{array}{ll}
0 & 0 \\
0 & 1
\end{array}\right], \quad D=\left[\begin{array}{cc}
1 & 0 \\
0 & -1
\end{array}\right], \quad D_{0}=\left[\begin{array}{cc}
-1 & 0 \\
0 & 0
\end{array}\right] .
\end{gathered}
$$

The polynomial system matrix corresponding to (24) is:

$$
P_{R P}\left(z_{1}, z_{2}\right)=\left[\begin{array}{cccc|cc}
z_{1} z_{2}-z_{1} & z_{1} & 0 & -1 & z_{1} & 0 \\
-2 z_{1} & z_{1} z_{2} & -2 & -1 & z_{1} & z_{1} \\
0 & 0 & z_{1}+1 & 0 & z_{1} & 0 \\
0 & -z_{1} & 0 & z_{1} & 0 & -z_{1} \\
\hline 0 & 0 & -1 & 0 & 0 & 0 \\
0 & 0 & 0 & -1 & 0 & 0
\end{array}\right]
$$

and the 2- $D$ transfer-function matrix is:

$$
G_{R P}\left(z_{1}, z_{2}\right)=\left[\begin{array}{cc}
\frac{z_{1}}{z_{1}+1} & 0 \\
\frac{z_{1}\left(z_{1} z_{2}+z_{1}+3 z_{2}-1\right)}{\left(z_{1}+1\right)\left(z_{1} z_{2}^{2}-z_{1} z_{2}+2 z_{1}-z_{2}-1\right)} & -\frac{z_{1}\left(z_{2}^{2}-2 z_{2}+3\right)}{z_{1} z_{2}^{2}-z_{1} z_{2}+2 z_{1}-z_{2}-1}
\end{array}\right]
$$




\section{Equivalence between discrete linear repetitive processes and the 2- $D$ singular general form}

Let $P_{R P}\left(z_{1}, z_{2}\right) \in \mathbb{R}^{[(n+m)+m] \times[(n+m)+p]}\left[z_{1}, z_{2}\right]$ be a $2-D$ polynomial system matrix given by (21). Then by introducing the state vector

$$
v(k, p)=\left[x_{k}^{T}(p) y_{k}^{T}(p)\right]^{T}
$$

$P_{R P}\left(z_{1}, z_{2}\right)$ is written in the $2-D$ singular form (11), i.e.,

$$
\tilde{P}_{R P}\left(z_{1}, z_{2}\right)=\left[\begin{array}{c|c}
z_{1} z_{2} \tilde{E}-z_{1} \tilde{A}_{1}-z_{2} \tilde{A}_{2}-A_{0} & z_{1} \tilde{B}_{1}+z_{2} \tilde{B}_{2}+\tilde{B}_{0} \\
\hline-\tilde{C} & \tilde{D}
\end{array}\right]
$$

where

$$
\begin{gathered}
\tilde{E}=\left[\begin{array}{cc}
I_{n} & 0 \\
0 & 0
\end{array}\right], \tilde{A}_{1}=\left[\begin{array}{cc}
A & 0 \\
C & -I_{m}
\end{array}\right], \tilde{A}_{2}=0, \tilde{A}_{0}=\left[\begin{array}{cc}
0 & B_{0} \\
0 & -D_{0}
\end{array}\right] \\
\tilde{B}_{1}=\left[\begin{array}{l}
B \\
D
\end{array}\right], \tilde{B}_{2}=\tilde{B}_{0}=0, \tilde{C}=\left[\begin{array}{ll}
0 & I_{m}
\end{array}\right], \tilde{D}=0 .
\end{gathered}
$$

Example 2 Consider the system matrix (25) corresponding to the discrete linear repetitive process given in Example 1 with 2- $D$ transfer-function matrix given by (26). Then using the system matrix in (28) the matrices $\tilde{A}_{i}, \tilde{B}_{i}, \tilde{C}$ and $\tilde{D}$ are given by:

$$
\begin{gathered}
\tilde{E}=\left[\begin{array}{llll}
1 & 0 & 0 & 0 \\
0 & 1 & 0 & 0 \\
0 & 0 & 0 & 0 \\
0 & 0 & 0 & 0
\end{array}\right], \tilde{A}_{1}=\left[\begin{array}{cccc}
1 & -1 & 0 & 0 \\
2 & 0 & 0 & 0 \\
0 & 0 & -1 & 0 \\
0 & 1 & 0 & -1
\end{array}\right], \tilde{A}_{2}=\left[\begin{array}{cccc}
0 & 0 & 0 & 0 \\
0 & 0 & 0 & 0 \\
0 & 0 & 0 & 0 \\
0 & 0 & 0 & 0
\end{array}\right], \\
\tilde{A}_{0}=\left[\begin{array}{cccc}
0 & 0 & 0 & 1 \\
0 & 0 & 2 & 1 \\
0 & 0 & -1 & 0 \\
0 & 0 & 0 & 0
\end{array}\right], \tilde{B}_{1}=\left[\begin{array}{cc}
1 & 0 \\
1 & 1 \\
1 & 0 \\
0 & -1
\end{array}\right], \tilde{B}_{2}=\tilde{B}_{0}=\left[\begin{array}{ll}
0 & 0 \\
0 & 0 \\
0 & 0 \\
0 & 0
\end{array}\right], \\
\tilde{C}=\left[\begin{array}{llll}
0 & 0 & 1 & 0 \\
0 & 0 & 0 & 1
\end{array}\right], \tilde{D}=\left[\begin{array}{ll}
0 & 0 \\
0 & 0
\end{array}\right] .
\end{gathered}
$$

\section{Reduction of a discrete linear repetitive process to the singular Roesser form}

Using the method given by Boudellioua (2012), the following result is obtained.

Theorem 1 Let $\tilde{P}_{R P}\left(z_{1}, z_{2}\right)$ be the $[(n+m)+m] \times[(n+m)+p]$ polynomial system matrix given by (28) and (29). Then $\tilde{P}_{R P}\left(z_{1}, z_{2}\right)$ is zero coprime system equivalent to a Roesser singular type system matrix of the form (15), i.e.,

$$
S_{1}\left(z_{1}, z_{2}\right) \tilde{P}_{R P}\left(z_{1}, z_{2}\right)=\tilde{P}_{S R}\left(z_{1}, z_{2}\right) S_{2}\left(z_{1}, z_{2}\right)
$$


where

$$
\tilde{P}_{S R}\left(z_{1}, z_{2}\right)=\left[\begin{array}{cccc|c}
I_{n+m} & -z_{1} I_{n+m} & 0_{n+m, p} & 0_{n+m, m} & 0_{n+m, p} \\
z_{2} \tilde{E}-\tilde{A}_{1} & -\tilde{A}_{0} & z_{1} \tilde{B}_{1} & 0_{n+m, m} & 0_{n+m, p} \\
0_{m, n+m} & -\tilde{C} & 0_{m, p} & I_{m} & 0_{m, p} \\
0_{p, n+m} & 0_{p, n+m} & -I_{p} & 0_{p, m} & I_{p} \\
\hline 0_{m, n+m} & 0_{m, n+m} & 0_{m, p} & -I_{m} & 0_{m, p}
\end{array}\right],
$$

and

$$
\begin{gathered}
S_{1}\left(z_{1}, z_{2}\right)=\left[\begin{array}{c|c}
0_{n+m, n+m} & 0_{n+m, m} \\
I_{n+m} & 0_{n+m, m} \\
0_{m, n+m} & 0_{m, m} \\
0_{p, n+m} & 0_{p, m} \\
\hline 0_{m, n+m} & I_{m}
\end{array}\right], \\
S_{2}\left(z_{1}, z_{2}\right)=\left[\begin{array}{c|c}
z_{1} I_{n+m} & 0_{n+m, p} \\
I_{n+m} & 0_{n+m, p} \\
0_{p, n+m} & I_{p} \\
\tilde{C} & 0_{m, p} \\
\hline 0_{p, n+m} & I_{p}
\end{array}\right] .
\end{gathered}
$$

Proof The transformation in (33) is in the required form and it remains to prove the equality (31) and the zero coprimeness of the matrices, where it is easily verified, given (29) and $\tilde{A}_{2}=0, \tilde{B}_{2}=\tilde{B}_{0}=0, \tilde{D}=0$, that the left and right-hand sides of (31) give

$$
\left[\begin{array}{cc}
0 & 0 \\
z_{1} z_{2} \tilde{E}-z_{1} \tilde{A}_{1}-\tilde{A}_{0} & z_{1} \tilde{B}_{1} \\
0 & 0 \\
0 & 0 \\
-\tilde{C} & 0
\end{array}\right]
$$

The zero left coprimeness of $\tilde{P}_{S R}\left(z_{1}, z_{2}\right)$ and $S_{1}\left(z_{1}, z_{2}\right)$ follows from the fact that the matrix $\left[\tilde{P}_{S R}\left(z_{1}, z_{2}\right) S_{1}\left(z_{1}, z_{2}\right)\right]$ has the highest order minor

$$
\operatorname{det}\left(\left[\begin{array}{ccccc}
I_{n+m} & 0 & 0 & 0 & 0 \\
z_{2} \tilde{E}-\tilde{A}_{1} & 0 & 0 & I_{n+m} & 0 \\
0 & I_{m} & 0 & 0 & 0 \\
0 & 0 & I_{p} & 0 & 0 \\
0 & -I_{m} & 0 & 0 & I_{m}
\end{array}\right]\right)= \pm 1
$$

obtained by deleting the second and third block columns. Similarly the zero right coprimeness of $\tilde{P}_{R P}\left(z_{1}, z_{2}\right)$ and $S_{2}\left(z_{1}, z_{2}\right)$ follows from the fact that the matrix $\left[\tilde{P}_{R P}^{T}\left(z_{1}, z_{2}\right) S_{2}^{T}\left(z_{1}, z_{2}\right)\right]^{T}$ has the highest order minor

$$
\operatorname{det}\left(\left[\begin{array}{cc}
I_{n+m} & 0 \\
0 & I_{p}
\end{array}\right]\right)=1
$$

obtained by deleting all the block rows except the fourth and the fifth. 
Remark 1 Note that in the commonly used notation for 2-D systems and linear repetitive processes the variables $z_{1}$ and $z_{2}$ are interchanged. Hence, the polynomial matrix of (33) refers to the singular Roesser model of the reversed form with polynomial matrix:

$$
P_{S R}\left(z_{1}, z_{2}\right)=\left[\begin{array}{c|c}
z_{2} E_{1}+z_{1} E_{2}-A & B \\
\hline-C & D
\end{array}\right],
$$

where the notation of (15) is adopted.

Example 3 Consider again the system matrix associated with the discrete linear repetitive process of Example 1. Then the corresponding system matrix $\tilde{P}_{S R}\left(z_{1}, z_{2}\right)$ in the 2-D singular Roesser form is:

$$
\tilde{P}_{S R}\left(z_{1}, z_{2}\right)=\left[\begin{array}{cccccccccccc|cc}
1 & 0 & 0 & 0 & -z_{1} & 0 & 0 & 0 & 0 & 0 & 0 & 0 & 0 & 0 \\
0 & 1 & 0 & 0 & 0 & -z_{1} & 0 & 0 & 0 & 0 & 0 & 0 & 0 & 0 \\
0 & 0 & 1 & 0 & 0 & 0 & -z_{1} & 0 & 0 & 0 & 0 & 0 & 0 & 0 \\
0 & 0 & 0 & 1 & 0 & 0 & 0 & -z_{1} & 0 & 0 & 0 & 0 & 0 & 0 \\
z_{2}-1 & 1 & 0 & 0 & 0 & 0 & 0 & -1 & z_{1} & 0 & 0 & 0 & 0 & 0 \\
-2 & z_{2} & 0 & 0 & 0 & 0 & -2 & -1 & z_{1} & z_{1} & 0 & 0 & 0 & 0 \\
0 & 0 & 1 & 0 & 0 & 0 & 1 & 0 & z_{1} & 0 & 0 & 0 & 0 & 0 \\
0 & -1 & 0 & 1 & 0 & 0 & 0 & 0 & 0 & -z_{1} & 0 & 0 & 0 & 0 \\
0 & 0 & 0 & 0 & 0 & 0 & -1 & 0 & 0 & 0 & 1 & 0 & 0 & 0 \\
0 & 0 & 0 & 0 & 0 & 0 & 0 & -1 & 0 & 0 & 0 & 1 & 0 & 0 \\
0 & 0 & 0 & 0 & 0 & 0 & 0 & 0 & -1 & 0 & 0 & 0 & 1 & 0 \\
0 & 0 & 0 & 0 & 0 & 0 & 0 & 0 & 0 & -1 & 0 & 0 & 0 & 1 \\
0 & 0 & 0 & 0 & 0 & 0 & 0 & 0 & 0 & 0 & -1 & 0 & 0 & 0 \\
0 & 0 & 0 & 0 & 0 & 0 & 0 & 0 & 0 & 0 & 0 & -1 & 0 & 0
\end{array}\right]
$$

It is routine to verify that $P_{R P}\left(z_{1}, z_{2}\right)$ and $\tilde{P}_{S R}\left(z_{1}, z_{2}\right)$ are related by the zero-coprime system equivalence transformation:

$$
\begin{aligned}
P_{R P}\left(z_{1}, z_{2}\right) S_{1}\left(z_{1}, z_{2}\right) & =S_{2}\left(z_{1}, z_{2}\right) \tilde{P}_{S R}\left(z_{1}, z_{2}\right) \\
& =\left[\begin{array}{cccccc}
0 & 0 & 0 & 0 & 0 & 0 \\
0 & 0 & 0 & 0 & 0 & 0 \\
0 & 0 & 0 & 0 & 0 & 0 \\
0 & 0 & 0 & 0 & 0 & 0 \\
z_{1}\left(z_{2}-1\right) & z_{1} & 0 & -1 & z_{1} & 0 \\
-2 z_{1} & z_{1} z_{2} & -2 & -1 & z_{1} & z_{1} \\
0 & 0 & z_{1}+1 & 0 & z_{1} & 0 \\
0 & -z_{1} & 0 & z_{1} & 0 & -z_{1} \\
0 & 0 & 0 & 0 & 0 & 0 \\
0 & 0 & 0 & 0 & 0 & 0 \\
0 & 0 & 0 & 0 & 0 & 0 \\
0 & 0 & 0 & 0 & 0 & 0 \\
0 & 0 & -1 & 0 & 0 & 0 \\
0 & 0 & 0 & -1 & 0 & 0
\end{array}\right]
\end{aligned}
$$


where

$$
S_{1}\left(z_{1}, z_{2}\right)=\left[\begin{array}{llll|ll}
0 & 0 & 0 & 0 & 0 & 0 \\
0 & 0 & 0 & 0 & 0 & 0 \\
0 & 0 & 0 & 0 & 0 & 0 \\
0 & 0 & 0 & 0 & 0 & 0 \\
1 & 0 & 0 & 0 & 0 & 0 \\
0 & 1 & 0 & 0 & 0 & 0 \\
0 & 0 & 1 & 0 & 0 & 0 \\
0 & 0 & 0 & 1 & 0 & 0 \\
0 & 0 & 0 & 0 & 0 & 0 \\
0 & 0 & 0 & 0 & 0 & 0 \\
0 & 0 & 0 & 0 & 0 & 0 \\
0 & 0 & 0 & 0 & 0 & 0 \\
0 & 0 & 0 & 0 & 1 & 0 \\
0 & 0 & 0 & 0 & 0 & 1
\end{array}\right], \quad S_{2}\left(z_{1}, z_{2}\right)=\left[\begin{array}{cccc|cc}
z_{1} & 0 & 0 & 0 & 0 & 0 \\
0 & z_{1} & 0 & 0 & 0 & 0 \\
0 & 0 & z_{1} & 0 & 0 & 0 \\
0 & 0 & 0 & z_{1} & 0 & 0 \\
1 & 0 & 0 & 0 & 0 & 0 \\
0 & 1 & 0 & 0 & 0 & 0 \\
0 & 0 & 1 & 0 & 0 & 0 \\
0 & 0 & 0 & 1 & 0 & 0 \\
0 & 0 & 0 & 0 & 1 & 0 \\
0 & 0 & 0 & 0 & 0 & 1 \\
0 & 0 & 1 & 0 & 0 & 0 \\
0 & 0 & 0 & 1 & 0 & 0 \\
\hline 0 & 0 & 0 & 0 & 1 & 0 \\
0 & 0 & 0 & 0 & 0 & 1
\end{array}\right]
$$

Furthermore the 2- $D$ transfer-function matrix corresponding to the system matrix $\tilde{P}_{S R}\left(z_{1}, z_{2}\right)$ of (38) is given by:

$$
\begin{aligned}
& G_{\tilde{S R}}\left(z_{1}, z_{2}\right)=\left[\begin{array}{cc}
\frac{z_{1}}{z_{1}+1} & 0 \\
\frac{z_{1}\left(z_{1} z_{2}+z_{1}+3 z_{2}-1\right)}{\left(z_{1}+1\right)\left(z_{1} z_{2}^{2}-z_{1} z_{2}+2 z_{1}-z_{2}-1\right)} & -\frac{z_{1}\left(z_{2}^{2}-2 z_{2}+3\right)}{z_{1} z_{2}^{2}-z_{1} z_{2}+2 z_{1}-z_{2}-1}
\end{array}\right] \\
& =G_{R P}\left(z_{1}, z_{2}\right)
\end{aligned}
$$

\section{Conclusions}

In this paper, equivalent representations are obtained in 2- $D$ general singular form and 2-D singular Roesser type form for a given system matrix arising from a discrete linear repetitive process. The exact connections between the original system matrix with its corresponding 2- $D$ singular forms have been developed and shown to be zero coprime system equivalence. The zero structures of the original polynomial system matrix are preserved, making it possible to analyze the polynomial system matrix in terms of its associated 2- $D$ singular forms.

One motivation for this work is that singular 2- $D$ representations are critical to examining certain physically well defined systems theoretic properties and, in particular, local controllability/reachability properties of discrete linear repetitive processes. It should also be possible to extend this analysis to other classes of linear repetitive processes. For example, in Rogers et al. (2007) it is established that cases exist where the pass state initial vector sequence is required to contain explicit terms from the previous pass profile, e.g.,

$$
x_{k+1}(0)=d_{k+1}+\sum_{j=1}^{M} \sum_{p=0}^{\alpha-1} K_{j p} y_{k+1-j}(p), \quad k \geq 0 .
$$

If the summation term is removed then the state initial vector sequence assumed in this paper results. However, under-modeling, e.g., assuming that this assumption can always be made is incorrect as the structure of the pass state initial vector sequence alone can destroy stability and also properties such as controllability/reachability. An example demonstrating this fact is given in Rogers et al. (2007).

The results in this paper show also the possibility of obtaining new equivalent forms of repetitive process description, which may have onward value in terms of the development of 
a comprehensive systems theory that is also supported by numerically reliable computational algorithms for checking systems theoretic properties, such as controllability, and the design of control laws. Possible future research could also include extending the analysis of this paper to more general forms of repetitive process dynamics.

Acknowledgements The authors wish to express their thanks to Sultan Qaboos University (Oman) for their support in carrying out this research work. Also, this work is partially supported by National Science Centre in Poland, Grant No. 2015/17/B/ST7/03703.

Open Access This article is distributed under the terms of the Creative Commons Attribution 4.0 International License (http://creativecommons.org/licenses/by/4.0/), which permits unrestricted use, distribution, and reproduction in any medium, provided you give appropriate credit to the original author(s) and the source, provide a link to the Creative Commons license, and indicate if changes were made.

\section{References}

Attasi, S. (1973). Systemes lineaires a deux indices. Technical Report 31. IRIA, France.

Boudellioua, M. S. (2012). Strict system equivalence of 2D linear discrete state space models. Journal of Control Science and Engineering, 2012 (Art. ID 609276), 6.

Fornasini, E., \& Marchesini, G. (1976). State space realization theory of two-dimensional filters. IEEE Transactions on Automatic Control, AC-21(4), 484-492.

Galkowski, K. (2001). State space realization of linear 2-D systems with extensions to the general $n D(n>2)$ case. London: Springer.

Galkowski, K., Rogers, E., \& Owens, D. H. (1998). Matrix rank based for reachabiltiy/controllability of discrete linear repetitive processes. Linear Algebra and its Applications, 275-276, 201-224.

Galkowski, K., Rogers, E., \& Owens, D. H. (1999). New 2D models and a transition matrix for discrete linear repetitive processes. International Journal of Control, 72(5), 1365-1380.

Johnson, D. S. (1993). Coprimeness in multidimensional system theory and symbolic computation. Ph.D. thesis, Loughborough University of Technology, UK.

Kaczorek, T. (1988). The singular general model of 2-D systems and its solution. IEEE Transactions on Automatic Control, 33(11), 1060-1061.

Levy, B. C. (1981). 2-D polynomial and rational matrices and their applications for the modelling of 2-D dynamical systems. Ph.D. thesis, Stanford University, USA.

Li, Xu, Yan, Shi, Lin, Zhiping, \& Matsushita, Shin ya. (2012). A new elementary operation approach to multidimensional realization and lfr uncertainty modeling: The MIMO case. IEEE Transactions on Circuits and Systems-I, 59(3), 638-651.

Pugh, A. C., \& El-Nabrawy, E. M. O. (2003). Zero structures of n-D systems. In Proceedings of the 11th mediterranean conference on control and automation. Rhodes, Greece.

Pugh, A. C., McInerney, S. J., Boudellioua, M. S., Johnson, D. S., \& Hayton, G. E. (1998). A transformation for 2-D linear systems and a generalization of a theorem of Rosenbrock. International Journal of Control, 71(3), 491-503.

Pugh, A. C., McInerney, S. J., Hou, M., \& Hayton, G. E. (1996). A transformation for 2-D systems and its invariants. In Proceedings of the 35th IEEE conference on decision and control (pp. 2157-2158). Kobe.

Roesser, R. P. (1975). A discrete state-space model for linear image processing. IEEE Transactions on Automatic Control, AC-20(1), 1-10.

Rogers, E., Galkowski, K., \& Owens, D. H. (2007). Control systems theory and applications for linear repetitive processes. Control and information sciences. Berlin, Heidelberg: Springer.

Rosenbrock, H. H. (1970). State space and multivariable theory. London, New York: Wiley.

Zak, S. H. (1984). On state-space models for sytems described by partial differential equations. In Proceedings of the 33rd IEEE conference on decision and control. Las Vegas. 